\title{
Influence of Regulation Logic on the Easiness of Evolving Sustained Oscillation for Gene Regulatory Networks
}

\author{
Yaochu Jin, Yan Meng, and Bernhard Sendhoff
}

\begin{abstract}
This paper investigates empirically the influence of regulation logic on the dynamics of two computational models of genetic regulatory network motifs. The gene regulatory network motifs considered in this work consist of three genes with both positive and negative feedback loops. Two forms of fuzzy logic, namely, the Zadeh operators and the probabilistic operators, as well as the summation logic have been investigated. We show that the easiness of evolving sustained oscillation, and the stability of the evolved oscillation depend both on the regulation logic and on the consistency of the regulation on the target gene.
\end{abstract}

\section{INTRODUCTION}

Modeling and analysis of gene regulatory networks is receiving increasing attention in computational systems biology. It has been found that a small number of sub-networks, also known as network motifs, occur very often in complex gene regulatory networks. These network motifs serve as building blocks of regulatory networks and the dynamics of the whole networks can be analyzed by analyzing these motifs. Detection and analysis of regulatory motifs in biological systems has now become one important research topic in systems biology [1], [2].

One line of fascinating research is to analyze the role of positive and negative feedback loops in the robustness and evolvability of gene regulatory networks. It has been found that negative feedback loops are a major mechanism for biological robustness, e.g., in heat shock response of $E$. Coli [3] and in perfect adaptation of bacteria chemotaxis [4]. A design principle found in cell signaling networks is that coherently coupled feedback loops are of essential importance to robustness [5], and that networks containing a large number of positive feedback loops and a small number of negative feedback loops are more likely to be robust to perturbations [6]. Most recently, it has been reported that a combination of positive feedback with negative feedback loops endows the networks with more robust and tunable sustained oscillations, and makes it easier to evolve stable oscillatory dynamics [7].

Meanwhile, regulatory control, particularly the regulation logic, is also attracting more and more research efforts. An experimental analysis of regulation control of the gene for development of the sea urchin has been conducted in [8]. A systematic investigation of control logic in gene regulation

Yaochu Jin and Bernhard Sendhoff are with the Honda Research Institute Europe, Carl-Legien-Str. 30, 63073 Offenbach, Germany (Email \{yaochu.jin, bernhard.sendhoff\}@honda-ri.de).

Yan Meng is with the Department of Electrical and Computer Engineering, Stevens Institute of Technology, NJ 07030, USA (Email: yan.meng@stevens.edu). has been performed in [9], which concludes that, among others, networks consisting of competitively binding activators and repressors can be controlled more robustly. Another interesting finding suggests that two types of logic control may exist in bacteria transcriptional networks, namely, a digital type and analog type [10]. Interestingly, these two distinct control types are found to be complementary in gene regulation. Negative feedback loops that promote systems robustness to mutations have also been shown to emerge in computational evolution of developmental system [11].

This paper investigates in silico the role of regulation logic in evolving oscillatory dynamics for two regulatory motifs consisting of a negative feedback loop and a positive feedback loop. To this end, we employ an evolution strategy, one of the widely used artificial evolutionary algorithms [12], to evolve the parameters of the given network motifs. Though evolution of the desired dynamics for a given network motif appears straightforward at the first sight, we find that it is nontrivial to evolve sustained oscillations, i.e., limit cycles. The most interesting finding from this work is that the easiness of evolving sustained oscillation depends not only on the regulation logic, but also on the way in which the feedback loops are connected to the target gene.

A few research efforts have been reported to evolve dynamics for gene regulatory networks in silico. In [13], both bistable switches and oscillators are evolved based on a number of predefined basic biochemical reactions. However, it was suggested in [14] that the results reported in [13] are not easily reproducible. In [14], a correlation based fitness function has been suggested, though no definite conclusion can be drawn on its influence on the successful evolution of oscillators. Similar work has also been reported in [15], where two different fitness functions are suggested for evolving oscillation. In [16], it is shown that a higher Hill co-efficient facilitates the evolution of sustained oscillation for the relaxation oscillator.

This paper is organized as follows. In Section II, a brief introduction to gene expression and the mathematical models of the studied network motifs are provided. The concept of regulation control is discussed in Section III, where a number of fuzzy logic expressions is also presented. Section IV describes very briefly the evolution strategy used in this work. Experimental results are given in Section $\mathrm{V}$ with discussions, and Section VI concludes the paper. 


\section{Regulatory Network Motifs and Regulation CONTROL LOGIC}

$\mathrm{h}$ The process of gene expression is composed of two main steps, namely, transcription of DNA to mRNA and translation of the mRNA to proteins. The expression of genes is controlled by biophysical and biochemical interactions among genes, proteins and metabolites. This network of interactions is termed the gene regulatory network.

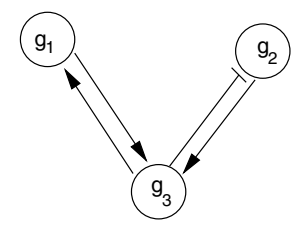

(a)

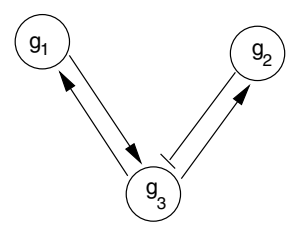

(b)
Fig. 1. Two regulatory motifs consisting of three gene, forming a negative feedback loop and a positive feedback loop. Gene $3\left(g_{3}\right)$ is the target gene. (a) Consistently regulated motif (CRM), (b) Inconsistently regulated motif (ICM).

We study two gene regulatory motifs, each consisting of three genes with a slight difference in connection, as shown in Fig. 1. Both motifs have a positive feedback loop and a negative feedback loop, namely, genes $g_{1}$ and $g_{3}$ formulate a positive feedback loop, while genes $g_{2}$ and $g_{3}$ build up a negative feedback loop. The only difference lies in the fact that in the consistently regulated motif (CRM) on the target gene $\left(g_{3}\right)$, refer to Fig. 1(a), meaning that both regulatory genes $\left(g_{1}\right.$ and $\left.g_{2}\right)$ are activating, and in the inconsistently regulated motif (IRM), see Fig. 1(b), $g_{1}$ activates the expression of $g_{3}$ whereas $g_{2}$ represses $g_{3}$. The mathematical model of the CRM can be described by the following differential equations:

$$
\begin{aligned}
& \dot{x}_{1}=a_{13} H_{13}\left(x_{3}\right)-a_{11} x_{1}, \\
& \dot{x}_{2}=a_{23} H_{23}\left(x_{3}\right)-a_{22} x_{2}, \\
& \dot{x}_{3}=a_{3} L\left(H_{31}\left(x_{1}\right), H_{32}\left(x_{2}\right)\right)-a_{33} x_{3},
\end{aligned}
$$

where $x_{i}, i=1,2,3$ are the concentration of the corresponding protein products of the three genes, $a_{11}, a_{22}$, and $a_{33}$ are the degradation rate of the proteins, and $a_{13}, a_{23}$, and $a_{3}$ are the parameters representing the strength of the protein interactions. All these parameters are non-negative, and

$$
\begin{aligned}
H_{13}\left(x_{3}\right) & =\frac{\beta x_{3}^{n}}{\theta_{1}^{n}+x_{3}^{n}}, \\
H_{31}\left(x_{1}\right) & =\frac{\beta x_{1}^{n}}{\theta_{3}^{n}+x_{1}^{n}}, \\
H_{23}\left(x_{3}\right) & =\frac{\beta}{1+\left(x_{3} / \theta_{2}\right)^{n}}, \\
H_{32}\left(x_{2}\right) & =\frac{\beta x_{2}^{n}}{\theta_{4}^{n}+x_{2}^{n}},
\end{aligned}
$$

where $\beta, \theta_{i}, i=1,2,3,4$, and $n$ are parameters in the activating and repressive Hill functions, where $n$ is called the Hill coefficient. We can see that $H_{13}, H_{31}$ and $H_{32}$ are activating, and $H_{23}$ is repressive.
For the IRM, everything is the same except that $H_{23}$ is now activating and $H_{32}$ is repressive:

$$
\begin{aligned}
H_{23}\left(x_{3}\right) & =\frac{\beta x_{3}^{n}}{\theta_{2}^{n}+x_{3}^{n}}, \\
H_{32}\left(x_{2}\right) & =\frac{\beta}{1+\left(x_{2} / \theta_{4}\right)^{n}} .
\end{aligned}
$$

In Equation (3), $L\left(H_{31}, H_{32}\right)$ is the function denoting the regulation logic that combines the influence of activating regulations from $g_{1}$ and $g_{2}$ of the expression of $g_{3}$. Often the case, various regulatory inputs are supposed to be additive. However, this may not be always true in biology, as discussed in [9]. Generally, different transcription factors can bind to the binding site of a gene either independently, competitively or cooperatively. In this work, we suppose $g_{1}$ and $g_{2}$ can bind to the binding site of $g_{3}$ independently. In addition, we consider the following two situations: 1) Both transcription factors produced by $g_{1}$ and $g_{2}$ are necessary for the expression of $g_{3}$, and 2) Either of the transcription factors will be sufficient for the expression of $g_{3}$. These two situations can be described by logic 'AND', logic 'OR', respectively. In the following section, we are going to introduce in more details the logic functions used in this work.

\section{FUZZY LOGIC}

Fuzzy logic systems have found a wide range of applications in science and engineering [17] since Zadeh's pioneering work on fuzzy sets [18] and fuzzy reasoning [19]. It is believed that fuzzy logic systems are particularly powerful in dealing with uncertainties in modeling, reasoning, and control, just to name a few. The unique ability of fuzzy systems can be attributed, in part, to the following two features. First, in contrast to the conventional set theory, an element either belongs to or does not belong to a set, while in the fuzzy set theory, an element may belong to a set with a degree between zero and one. This membership degree is defined by a piece-wise continuous membership function whose value is between 0 and 1 . Second, the fuzzy logic operators that allow for more flexible processing of information. In the earlier age, most fuzzy systems were built upon human heuristics, or from observations of human experts. Since the beginning of 1990's, data-driven fuzzy systems have been playing an increasingly important role, where fuzzy rules are abstracted from experimental data. One main new feature of the data-driven fuzzy systems is their ability to learn, which can be largely attributed to the marriage of machine learning techniques, such as neural networks and evolutionary algorithms with fuzzy set theory [20], [21], [22].

This work investigates the role of fuzzy logic in modeling gene regulation control and its relationship to evolving oscillatory dynamics. In gene regulation, the expression of a gene is often regulated by a number of regulatory units (enhancers or silencers), and more than one transcription factor can be bound to a binding site. The question is, does it need all activating TFs to activate the expression of the gene, or is it sufficient to have only one of the TF to activate? Another 
question is, if multiple TFs can be bound to the binding site, are they independent, competitive, or as a complex only?

In this work, we try to answer the first question mentioned above to a certain degree. As shown in Fig. 1, the regulatory motif we are studying contains three genes, where gene 3 is activated by both gene 1 and gene 2. Two possibilities are considered in the following: 1) Genes 1 and 2 are both needed to activate gene 3 , which can be modeled using the fuzzy 'AND' logic; 2) Either gene 1 or gene 2 are needed to activate gene 3 , modeled using the fuzzy 'OR' logic.

Two types of fuzzy logic formulations are investigated in the simulations. The first type is the Zadeh operators:

$$
\begin{aligned}
\text { AND: } & x \bigwedge y=\min (x, y), \\
\text { OR: } & x \bigvee y=\max (x, y),
\end{aligned}
$$

where ' $\Lambda$ ' and ' $\bigvee$ ' denote fuzzy 'AND' and 'OR', respectively, $\min (x, y)$ and $\max (x, y)$ return the minimum and the maximum of $x$ and $y$, respectively.

The second type of fuzzy logic operators is known as the probabilistic operators, which can be described as follows:

$$
\begin{aligned}
\text { AND: } & x \bigwedge y=x y \\
\text { OR: } & x \bigvee y=x+y-x y
\end{aligned}
$$

In addition to the above fuzzy logic operations, we also investigate the summation logic, which is used in most gene regulatory network models. In summary, the following five logic operators have been investigated for the regulation logic:

$$
\begin{aligned}
\text { Zadeh 'AND': } & L(x, y)=\min (x, y), \\
\text { probabilistic 'AND': } & L(x, y)=x y, \\
\text { Zadeh 'OR': } & L(x, y)=\max (x, y), \\
\text { probabilistic 'OR': } & L(x, y)=x+y-x y, \\
\text { summation: } & L(x, y)=\frac{1}{2}(x+y) .
\end{aligned}
$$

It is interesting to note that summation can be considered as a linear combination of the probabilistic 'AND and the probabilistic 'OR'. It should also be noticed that in fuzzy logic operations, the value of $x$ and $y$ is always limited between zero and one. In this work, we require that the value is non-negative, but it is allowed to be larger than one.

\section{Evolution Strategy}

Evolution strategies are one of the widely used artificial evolutionary algorithms that are very effective for optimizing real-valued problems. Since the structure of the regulatory motifs is fixed, and only the parameters are evolved in this work, we adopt a canonical evolution strategy for evolving the desired dynamics. In a canonical evolution strategy (ES), the mutation of the object parameters (the parameters to be optimized) is performed by adding an $N\left(0, \sigma_{i}^{2}\right)$ distributed random number, where $\sigma_{i}$ 's are termed as strategy parameters that are also encoded in the genotype and subject to mutations. The ES used in this work can be described as follows:

$$
\begin{aligned}
\mathbf{x}(t) & =\mathbf{x}(t-1)+\tilde{\mathbf{z}} \\
\sigma_{i}(t) & =\sigma_{i}(t-1) \exp \left(\tau^{\prime} z\right) \exp \left(\tau z_{i}\right) ; i=1, \ldots, n,
\end{aligned}
$$

where $\mathbf{x}$ is an $n$-dimensional parameter vector to be evolved, $\tilde{\mathbf{z}}$ is an $n$-dimensional random number vector with $\tilde{\mathbf{z}} \sim$ $N\left(\mathbf{0}, \sigma(t)^{2}\right), z$ and $z_{i}$ are normally distributed random numbers with $z, z_{i} \sim N(0,1)$. Parameters $\tau, \tau^{\prime}$ and $\sigma_{i}$ are the strategy parameters, also known as step-sizes, where $\sigma_{i}$ is mutated as in equation (22) and $\tau, \tau^{\prime}$ are constants as follows:

$$
\tau=(\sqrt{2 \sqrt{n}})^{-1} ; \tau^{\prime}=(\sqrt{2 n})^{-1} .
$$

Two selection schemes have been proposed in evolution strategies, known as comma and plus strategies. Suppose there are $\mu$ and $\lambda$ individuals in the parent and offspring population, usually $\mu \leq \lambda$. In the comma strategy, $\mu$ parent individuals are selected only from the $\lambda$ offspring individuals, which is usually noted as $(\mu, \lambda)$-ES. In the plus strategy, $\mu$ parent individuals are selected from a union of $\mu$ parent individuals and $\lambda$ offspring individuals, which is noted as $(\mu+\lambda)$-ES. In our study, the $(\mu, \lambda)$-ES is adopted.

In the evolution, all parameters in the regulatory model, i.e., three decay rates and three synthesis rates, one coefficient $(\beta)$, four thresholds, and one Hill coefficient are the object parameters encoded in the genome.

\section{Simulation Results}

A (30, 200)-ES has been adopted in our experiments. All object parameters to be evolved are randomly initialized between 0 and 4 . According to the physical meaning of the parameters, a lower bound is set to 0 for all parameters, but no upper bound is given. The initial step-size is set to 1 . In all simulations, 500 generations are run for each case.

The aim of the work is to produce a sustained oscillatory dynamics for the concentration of $g_{3}$. The target function for $x_{3}$ in evolving oscillation is defined by a sinus function as follows:

$$
x_{3}^{d}(t)=\sin (2 \pi t / \mathrm{T})+1.0,
$$

where $t$ is time instant, and $T$ is the desired period of the oscillation. The fitness function of the evolution strategy is to minimize the following error:

$$
E=\sum_{t=0}^{N}\left(x_{3}(t)-x_{3}^{d}(t)\right)^{2},
$$

where $x_{3}(t)$ is the real output, $N$ is the time steps considered in evolution. The differential equations are solved using the Euler method. The time interval for the Euler solver is set to 0.1 and $N$ is set to 200 in this work. In the simulations, a desired period of $T=1,2, \ldots, 12$ is chosen in 12 groups of simulations for each motif, and for each desired period, 10 independent runs are performed. Note that during the evolution, $x_{1}$ and $x_{2}$ are initialized to 1.0 , while $x_{3}$ is initialized to 0 . 


\section{A. Easiness of Evolving Sustained Oscillation}

Fig. 2 shows the percentage of successful evolution of sustained oscillation in 10 independent runs for each desired period, when different logic functions are employed for both CRM and IRM. An evolution is considered successful if the evolved output produces a stable oscillation. The oscillation is said to be stable if the change in frequency and amplitude is smaller than a given threshold within 400 time steps. For CRM, when the probabilistic 'AND' operator is used, the system is able to evolve sustained oscillation for 16 times from a total of 120 independent runs. When the probabilistic 'OR', or summation is used, sustained oscillation is able to evolve only three times in 120 runs. When Zadeh 'AND' or Zadeh 'OR' is used for combining different regulatory elements, none of the runs has been successful in evolving sustained oscillation. Different to the IRM, the highest percentage (29 times in 120 runs) of successful evolution of limited cycles is achieved for CRM when summation is adopted as the regulation logic. When probabilistic 'OR' is used, the system is able to evolve limit cycles in 15 cases out of 120 runs. Similar to CRM, no successful runs have been achieved in evolving limit cycles. More empirical and theoretic investigations are required to clarify why it fails to evolve limit cycles when the Zadeh operators are used.

Although the successful rates are quite low in general, it is interesting to notice that a large Hill coefficient is not required, which is different to the findings in [16], where relaxation oscillator using the sum logic was studied.

\section{B. Period and Amplitude of the Limit Cycles}

1) Accuracy in Learning the Desired Period: We first compare the period of the evolved limit cycles with that of the desired. The relationship between the desired and evolved periods are plotted in Fig. 3 (a) for CRM, and in Fig. 3 (b) for IRM. In general, the period of the evolved limit cycles is close to that of the desired in all cases in which a limit cycle has successfully evolved. To be exact, the mean absolute approximation errors are $0.24,0.6$, and 0.45 for CRM, and 0.2 and 0.37 for IRM, respectively. Note that the simulation interval is 0.1 , i.e., the temporal resolution of the simulation is 0.1 .

2) Frequency-Amplitude Relation: It has been observed that it is of great importance in biology that a regulatory system can produce different oscillation frequencies with a similar oscillation amplitude, where the positive feedback loop helps keep the amplitude relatively unchanged [7]. Since the regulatory motifs we studied in this work also contain a negative feedback loop plus a positive one, we are interested in investigating how amplitude changes as the frequency (or period) of the oscillation changes. For this purpose, the relationship between the evolved period and the amplitude of the limit cycles are presented in Fig. 4(a) for the IRM, and Fig. 4(b), respectively. The evolved frequency is estimated in 400 time steps. It seems that for the CRM, the amplitude is rather stable when summation is used for the regulation logic.

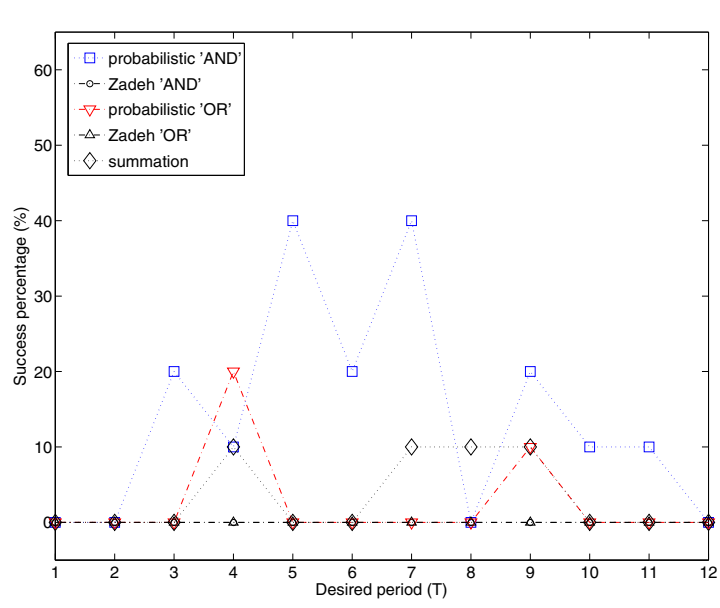

(a)

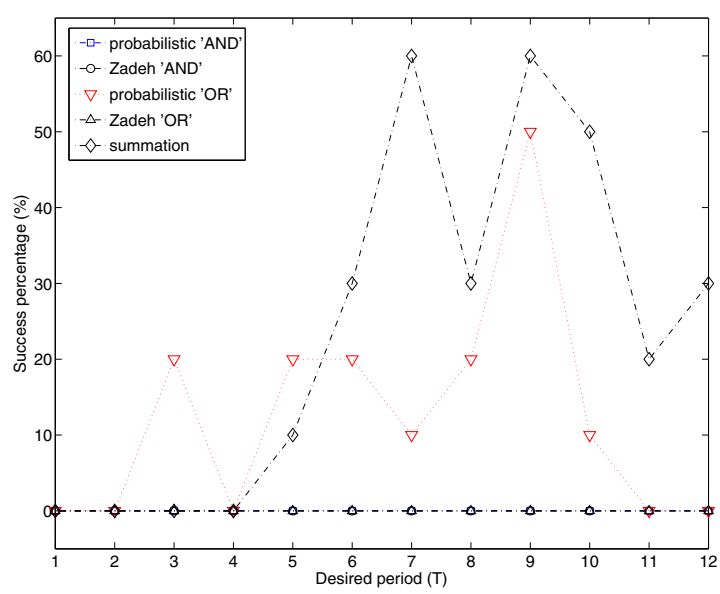

(b)

Fig. 2. Success percentage in evolving sustained oscillation. (a) CRM, (b) IRM.

\section{Sensitivity of the Evolved Limit Cycles}

We now present a few typical examples of the evolved limit cycles, as shown in Figs. 5-8 for IRM with sum, probabilistic 'AND' as the regulation logic, and CRM with sum and probabilistic 'OR' as the regulation logic, respectively. In the four figures, the left panel shows the trajectories starting from 50 random initial points in the state space, and the right panel the profile of the $x_{3}$ (concentration of $g_{3}$ ) over time. From these dynamics, we have observed that the limit cycles generated for the CRM using probabilistic operator as the regulation logic is quite sensitive to the initial system state. Some of the initial states lead to an equilibrium, and the amplitude of the limit cycles are most often dependent on the initial states, refer to Fig. 5. However, the limit cycles evolved for the IRM using summation is insensitive to the initial system states, see e.g., Fig. 6. In contrast, all limit cycles generated by the evolved CRM are robust to the initialization of the system state. 


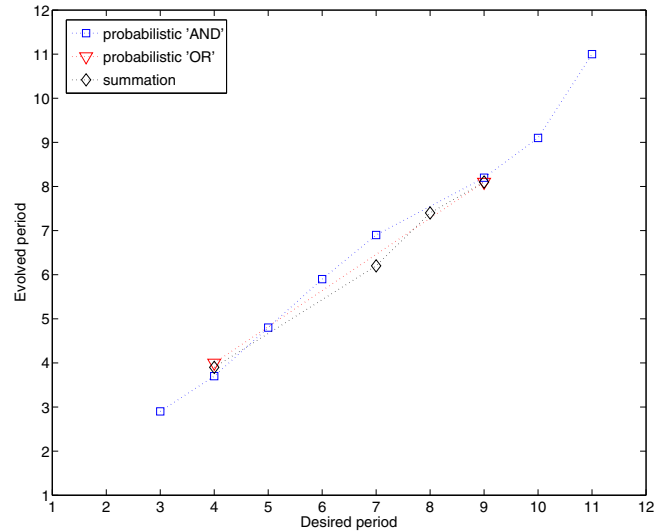

(a)

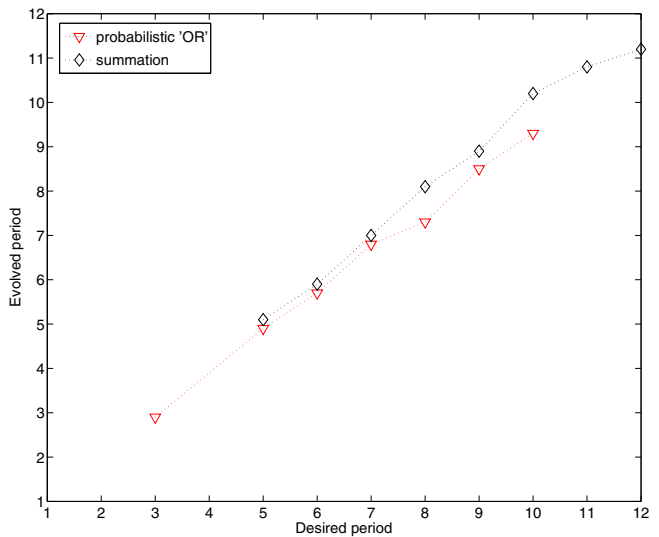

(b)

Fig. 3. Evolved periods compared to the desired ones. (a) CRM, and (b) IRM.

\section{Illustrative Examples of Regulatory Dynamics}

In this subsection, we illustrate a few evolved regulatory dynamics including sustained oscillations, damped oscillation and bi-stability, which may be of biological implication.

1) Limit cycle together with a stable equilibrium: As mentioned in Section V-C, one interesting difference between the dynamics of CRM and IRM that exhibit sustained oscillation is that the oscillation dynamics of the CRM is sensitive to the initial states. For CRM with the probabilistic 'AND', 15 of the 16 evolved sustained oscillation is subject to the initial states of the system. For CRM with probabilistic 'OR' logic, two of the three evolved limit cycle are subject to the initial states. In other word, sustained oscillation appears only for part of the initial states, when the range of the initial states tested is set to $[0,4]$ for all the three variables. Fig. 9 shows an example with probabilistic 'AND' and a desired period $T=5$, while Fig. 10) an example with a probabilistic 'OR' and a desired period $T=4$.

2) Bi-stability: Although the desired dynamics is a sustained oscillation, bi-stable dynamics often emerges in the

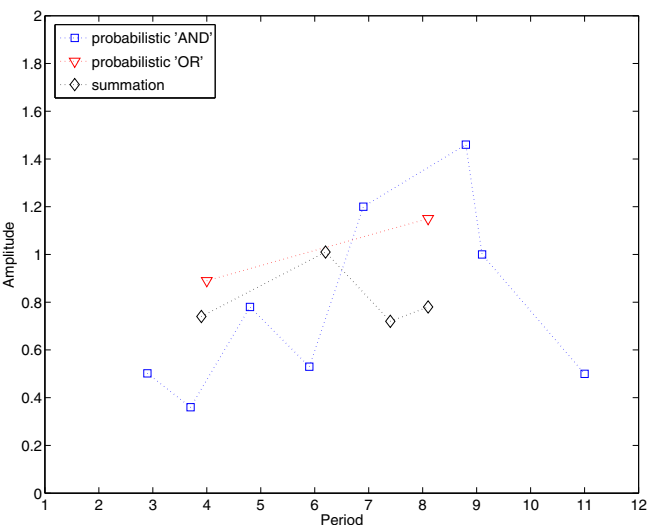

(a)

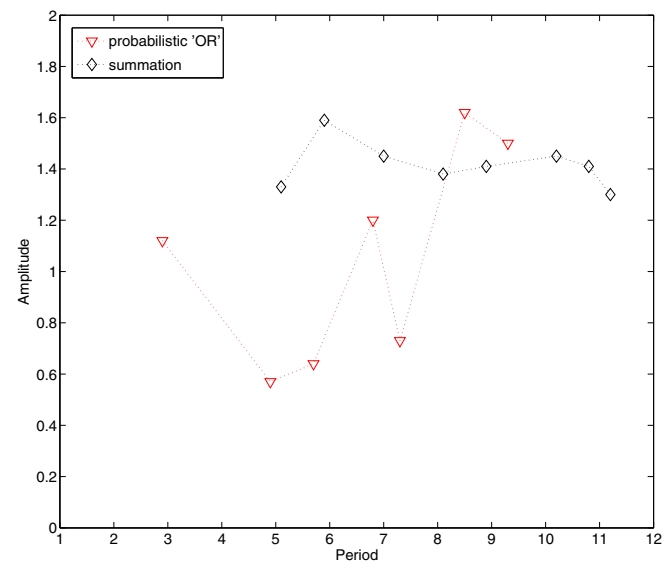

(b)

Fig. 4. Amplitude and period of the evolved limit cycles. (a) CRM, (b) IRM.

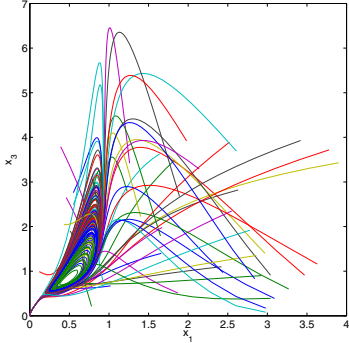

(a)

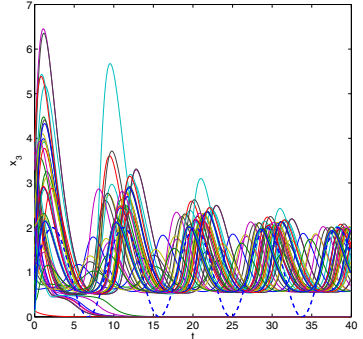

(b)
Fig. 5. CRM with probabilistic 'AND' as the regulation logic, $T=9$. (a) Trajectories of 50 random inital states in the state space. (b) Time course of target gene $\left(x_{3}\right)$. The thick dashed line denotes the desired signal. 


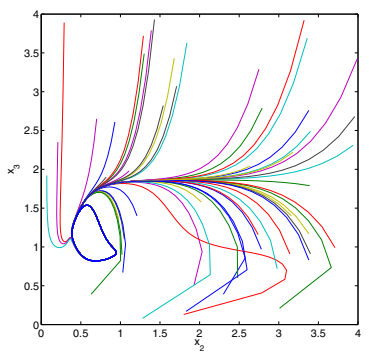

(a)

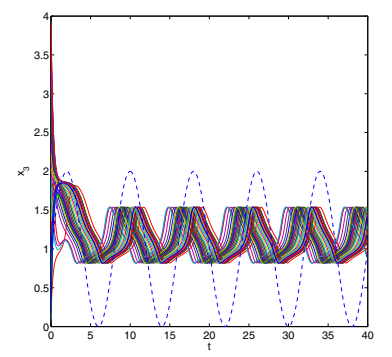

(b)

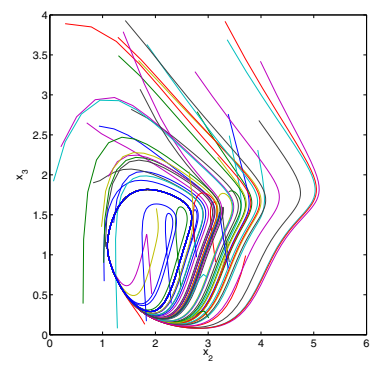

(a)

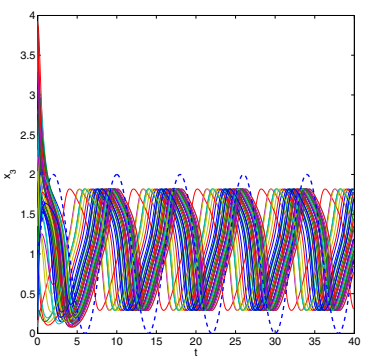

(b)
Fig. 6. CRM with sum as the regulation logic, $T=8$. (a) Trajectories of 50 random initial states in the state space. (b) Time course of target gene $\left(x_{3}\right)$. The thick dashed line denotes the desired signal.

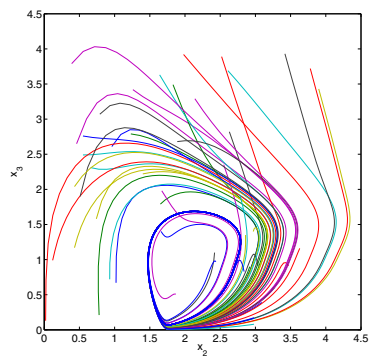

(a)

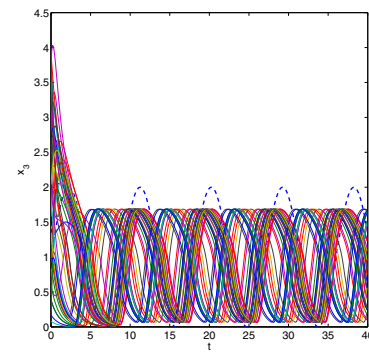

(b)
Fig. 7. IRM with probabilistic 'OR' as the regulation logic, $T=9$. (a) Trajectories of 50 random initial states in the state space. (b) Time course of target gene $\left(x_{3}\right)$. The thick dashed line denotes the desired signal.

evolved systems, particularly when the probabilistic 'OR' logic is used. Two kinds of bi-stable dynamics have been generated, either one oscillatory attractor and an equilibrium as shown in Fig. 11(a), or two equilibria, see Fig. 12(b).

3) An unstable attractor plus an equilibrium: For both CRM and IRM, interesting dynamics such as an unstable attractor (UA) plus an equilibrium has been observed. This means that the system first approaches an attractor and then converges to a stable equilibrium, see Fig. 12 for the CRM and Fig. 13 for the IRM, where the unstable attractor is denoted with UA.

\section{E. Discussions}

The biological meanings of the dynamics evolved for the regulatory motifs in this work remains to be revealed. On the one hand, we show that rich dynamics, such as limit cycles, attractors, equilibria, as well as bistability, has been evolved successfully for very simple regulatory motifs, which indicates that the systems ability to generate rich phenotypic features. On the other hand, the biological implication of such richness in dynamics, particularly its role in biological evolution, is still unclear.

\section{CONCLUSiOnS}

This paper reports our initial results on the influence of regulation logic on the easiness of evolving oscillatory
Fig. 8. IRM with sum as the regulation logic, $T=8$. (a) Trajectories of 50 random initial states in the state space. (b) Time course of target gene $\left(x_{3}\right)$. The thick dashed line denotes the desired signal.

dynamics for gene regulatory motifs. Three interesting phenomena have been observed. First, the easiness of evolving sustained oscillation depends not only on the regulation logic, but also on the consistency of the regulation on the target gene. Second, both for CRM and IRM, the desired period can be evolved with a relatively good accuracy. Third, the stability of the evolved oscillations may depend on the initial state of the system, and the sustained oscillation of the CRM seems to be less sensitive to initial states.

A few interesting issues remain to be investigated. For example, if regulation logic is not predefined, will one that leads to more robust oscillation emerge? Similarly, if the connectivity of the regulatory motifs is not given, is the evolution able to find a structure that can produce more robust oscillation? An answer to these questions will hopefully help us understand the occurrence of the network motifs found in biology.

\section{REFERENCES}

[1] U. Alon, An Introduction to Systems Biology: Design Principles of Biological Circuits. Boca Raton: Chapman \& Hall, 2007.

[2] —_ "Network motifs: theory and experimental approaches," Nature Review Genetics, vol. 8, pp. 450-461, 2007.

[3] H. El-Samad, H. Kurata, J. Doyle, C. Gross, and M. Khammash, "Surviving heat shock: Control strategies for robustness and performance," Proceedings of the National Academy of Science of the USA, vol. 102, no. 8, pp. 2736-2741, 2005.

[4] T.-M. Yi, Y. Huang, M. Simon, and J. Doyle, "Robust perfect adaptation in bacterial chemotaxis through integral feedback control," Proceedings of the National Academy of Science of the USA, vol. 97, no. 9, pp. 4649-4653, 2000.

[5] Y.-K. Kwon and K.-H. Cho, "Co-herent coupling of feedback loops: A design principle of cell signalling networks," Bioinformatics, vol. 24, no. 17, pp. 1926-1932, 2008.

[6] — "Quantitative analysis of robustness and fragility in biological networks based on feedback dynamics," Bioinformatics, vol. 24, no. 7 , pp. 987-994, 2008.

[7] T. Y.-C. Tsai, Y. Choi, W. Ma, J. Pomerening, C. Tang, and J. Ferrel Jr., "Robust, tunable biological oscillations from interlinked positive and negative feedback loops," Science, vol. 321, pp. 126-129, 2008.

[8] C.-H. Yuh, H. Bolouri, J. Bower, and E. Davidson, "A logical model of cis-regulatory control in a Eukatyotic system," in Computational Modeling of Genetic and Biochemical Networks, J. Bower and H. Bolouri, Eds. The MIT Press, 2000, pp. 73-100.

[9] M. Schilstra and C. Nehaniv, "Bio-logic: Gene expression and the laws of combinatorial logic," Artificial Life, vol. 14, pp. 121-133, 2008. 


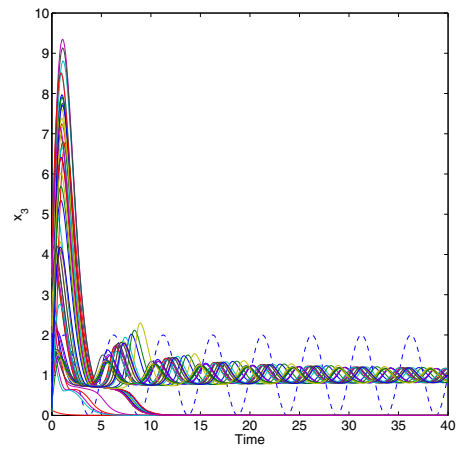

(a)

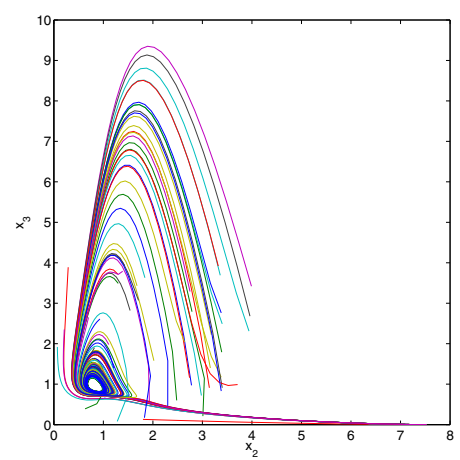

(b)

Fig. 9. CRM with probabilistic 'AND'. (a) Time course, and (b) state-space trajectory of 50 random initial states.

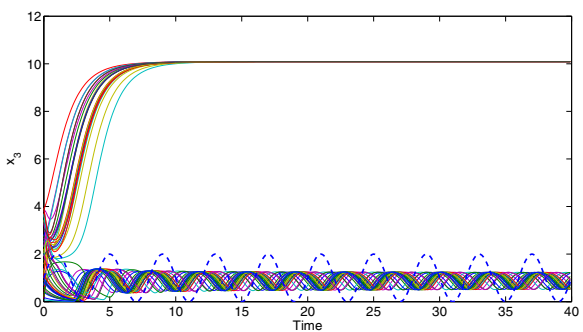

(a)

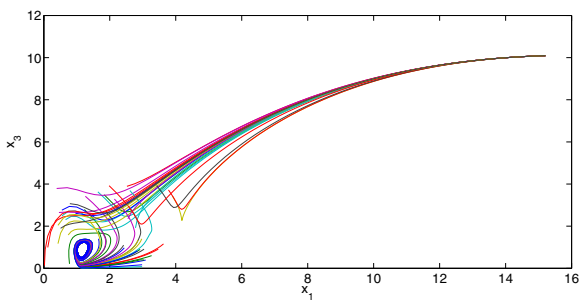

(b)

Fig. 10. CRM with probabilistic 'OR'. (a) Time course, and (b) state-space trajectory of 50 random initial states.

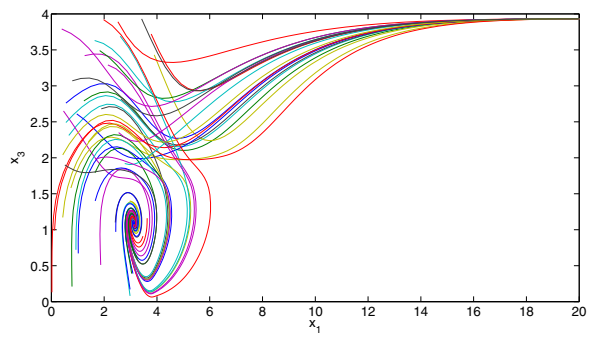

(a)

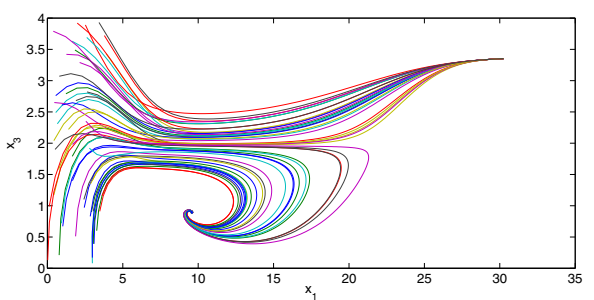

(b)

Fig. 11. Bi-stable dynamics from two evolved CRMs with probabilistic 'OR' logic. (a) $T=6$, and (b) $T=12$.

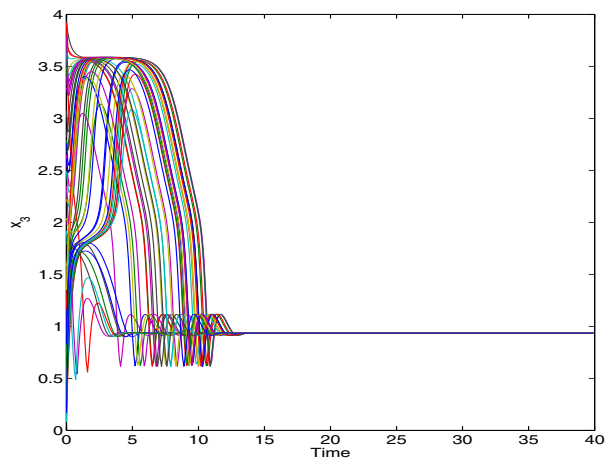

(a)

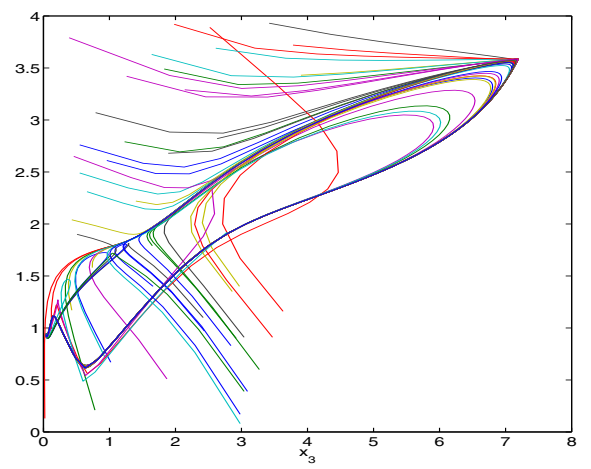

(b)

Fig. 12. Dynamics of an evolved CRM with summation when the desired period $T=12$. (a) Time course, (b) state-space trajectory. 


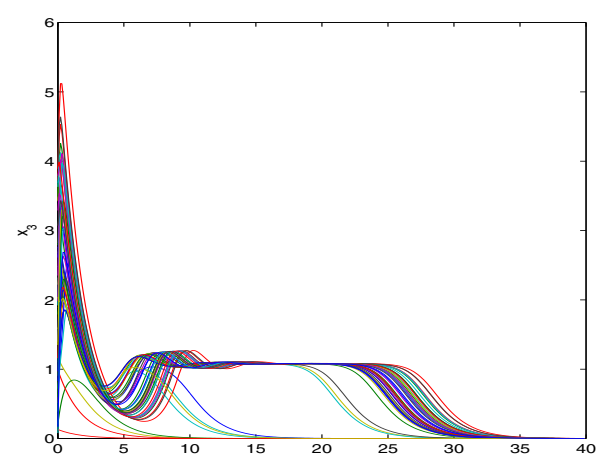

(a)

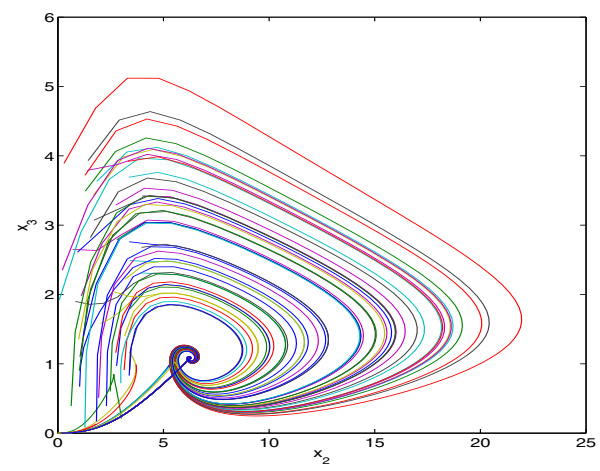

(b)

Fig. 13. Dynamics of an evolved IRM with Zadeh 'AND' when the desired period $T=6$. (a) Time course, (b) state-space trajectory.

[10] C. Marr, M. Geertz, M.-T. Hütt, and G. Muskhelishvili, "Dissecting the logic of network control in gene expression profiles," BMC Systems Biology, vol. 2, p. 18, 2008.

[11] T. Steiner, L. Schramm, Y. Jin, and B. Sendhoff, "Emergence of feedback in artificial gene regulatory networks." in Congress on Evolutionary Computation, 2007, pp. 867-874.

[12] T. Bäck, Evolutionary Algorithms in Theory and Practice: Evolution Strategies, Evolutionary Programming, Genetic Algorithms. Oxford University Press, 1996.

[13] P. Francois and V. Hakim, "Design of genetic networks with specified functions by evolution in silico," Proceedings of the National Academy of Sciences of USA, vol. 101, no. 2, pp. 580-585, 2007.

[14] D. Chu, "Evolving genetic regulatory networks for systems biology," in Congress on Evolutionary Computation. IEEE Press, 2007, pp. 875-882.

[15] S. Paladugu, V. Chickarmane, A. Dekard, J. Frumkin, M. MaCormarck, and H. Sauro, "In silico evolution of functional modules in biochemical networks," IEEE Proceedings on Systems Biology, vol. 153, no. 4, pp. 223-235, 2006.

[16] Y. Jin and B. Sendhoff, "Evolving in silico bistable and oscillatory dynamics for gene regulatory network motifs," in Congress on Evolutionary Computation, 2008, pp. 386-391.

[17] G. Klir, U. Clair, and B. Yuan, Fuzzy Set Theory: Foundations and Applications. Prentice Hall, 1997.

[18] L. Zadeh, "Fuzzy sets," Information Control, vol. 8, no. 3, pp. 338 353, 1965.

[19] — "Outline of a new approach to the analysis of complex systems and decision processes," IEEE Transactions on Systems, Man, and Cybernetics, vol. 3, no. 1, pp. 28-44, 1971.

[20] O. Cordon, F. Herrera, F. Hoffmann, and L. Magdalena, Genetic Fuzzy Systems - Evolutionary Tuning and Learning of Fuzzy Knowledge Bases. Singapore: World Scientific, 2001.
[21] J.-S. R. Jang, C.-T. Lin, and E. Mitzutani, Neuro-Fuzzy and Soft Computing. Pretice Hall, 1997.

[22] Y. Jin, Advanced Fuzzy Systems Design and Applications. Heidelberg: Physica/Springer, 2003. 